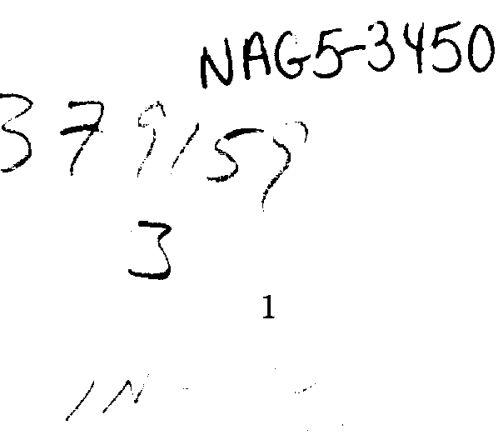

\title{
Granularity of the Diffuse Background Observed
}

\author{
D. E. Gruber ${ }^{a *}$, D. MacDonald ${ }^{a}$ R. E Rothschild ${ }^{a}$, E. Boldt ${ }^{b}$, R. F. Mushotzky ${ }^{b}$, and A. C. Fabian ${ }^{c}$ \\ aCenter for Astrophysics and Space Sciences, University of California, San Diego, \\ 9500 Gilman Dr., La Jolla, CA, 92093-0424, U. S. A. \\ ${ }^{\mathrm{b}}$ Goddard Space Flight Center, Greenbelt, MD 20771, U. S. A. \\ 'Institute of Astronomy, University of Cambridge, \\ Madingly Road, Cambridge CB3 0HA, United Kingdom
}

First results are reported from a program for measuring the field-to-field fluctuation level of the cosmic diffuse background by using differences between the two background positions of each deep exposure with the HEXTE instrument on RXTE. With 8 million live seconds accumulated to date a fluctuation level on the 15-25 keV band is observed which is consistent with extrapolations from the HEAO-1 measurements. Positive results are expected eventually at higher energies. Models of AGN origin will eventually be constrained by this program.

\section{INTRODUCTION}

The origin of the X-ray diffuse background continues to be a challenging problem. The background intensity is still best explained as the sum of emission from point sources (e. g. [1]). Near 1 $\mathrm{keV}$, source counts from deep x-ray imaged fields have regularly arrived at the tantalizing value of roughly $50 \%$ contribution to the total. This lack of closure is discomforting, but the greatest challenge to the superposition-of-sources origin comes in fact at higher energies, particularly in the interval $2-40 \mathrm{keV}$, where the flat background spectrum is completely unlike that of any ensemble of known extragalactic sources. Subtraction of the contribution due to known sources only makes the situation worse.

In recent years, however, steady advances toward resolution of this "spectral paradox" have been made both in theory and observation: the identification of the Compton reflection mechanism [2] showed how a flat spectral component could arise in the context of a centralengine active galaxy model; combined observations from GINGA $[3,4]$ first indicated the presence of this component in AGN. However, the observed strength of the reflection component in

*We acknowledge support from NASA grants NAG5-3450 and NAG5-4798 the low redshift AGN is not strong enough to account for the x-ray background. Another possible algebraic decomposition of the x-ray background [5] consists of a sum of relatively unabsorbed AGN and highly absorbed objects. This model is consistent with unified theories of AGN in which Seyfert 2 galaxies contain a generic AGN engine seen through a molecular torus, while unabsorbed objects correspond to Seyfert 1's. Very recently ASCA has provided us with examples of such highly absorbed AGN with columns of greater than $10^{23} \mathrm{~cm}^{2}$ : NGC4945 [6], M106 [7] and NGC6552 [8]. All models predict that the observed X-ray background $\log \mathrm{N}-\log \mathrm{S}$ at high energies has a relatively higher normalization than at lower energies, as required by a comparison of the HEAO-1 A2/GINGA fluctuation analysis in the $2-10 \mathrm{keV}$ (e.g. [9]) with the Einstein/Rosat soft $x$-ray $\log N-\log S$ and tentative results from the HEAO-1 A4 data (Boldt, reported by Gruber [10]). In this scenario the spectrum of faint, hard $\mathrm{x}$-ray selected objects should show the presence of either very strong reflection or of strong absorption. While a catalog of such objects appropriate for observing with XTE does not exist, the spectrum of the fluctuations in the x-ray background, which are due to the fainter unresolved objects, should have such a signature. XTE is the first 
mission capable of measuring the amplitude of the fluctuations at $\mathrm{E}>10 \mathrm{keV}$ and of determining the spectrum of the fluctuations to about 60 $\mathrm{keV}$.

In spite of the recent progress sketched above, there are still numerous points of difficulty (e.g. [11]) in relating individual and collective source measurements with even the overall background properties. The steep OSSE spectra of AGN [12] indicate a break in the range, $40-100 \mathrm{keV}$, which can be sensitively measured by HEXTE with long exposures. However a re-analysis of the OSSE data [13] concludes that such a break is not a necessary consequence of the OSSE data if one includes the effects of reflection. Zdziarski et al. [12] discuss whether present epoch AGN's with such a break can be used as a satisfactory basis for a spectral model of the background.

Long exposures of extragalactic objects are part of the XTE observing program. What we have undertaken here is the continued use of the HEXTE off-source background data from otherwise-scheduled observations to collect statistical information about even fainter extragalactic sources. By taking differences of each cluster's two independent background positions we measure the fluctuation level, or granularity, of the diffuse background as a function of energy on the range $12-60 \mathrm{keV}$. Above $60 \mathrm{keV}$ we expect counting statistics to dominate sky fluctuations.

\section{SENSITIVITY}

Sensitivity calculations are based on extrapolation of the fluctuation level measured with HEAO-1 A2 [14] to the diffuse flux above 12 $\mathrm{keV}[15,10]$ and to the area and field of the HEXTE, and allowing for the observed counting rates and exposures in the first XTE observing round (AO1) of 9 months duration. We define two energy bands, one below and one above a strong internal background line at $30 \mathrm{keV}$. A simple calculation indicates that in a single 100,000 second observation the sky fluctuation level just exceeds counting statistics in the lower $15-25 \mathrm{keV}$ band. Given 840 fluctuation measurements from 420 sources, 60 from AO1 and 180 each from the later one-year observing rounds $\mathrm{AO} 2$ and $\mathrm{A} 03$, the net fluctuation, or P(D) distribution can yield a fluctuation level determination on each band and a two-point spectrum from which one can infer the average spectrum of sources at $\mathrm{z}=0.3$. Outliers in this $\mathrm{P}(\mathrm{D})$ distribution suggest serendipitous sources at the level of about 0.1 count per second, roughly a millicrab, which can be detected at lower energies in about 5 minutes by the PCA.

The A01 results have been encouraging. With the AO1 observations completed, 8 million seconds of live time have been received and processed, from which we presently have a $2 \sigma$ detection of the fluctuations in the XRB in excess of the fluctuations due to counting statistics of our ensemble of measurements and a peak probability at a fluctuation level slightly higher than our extrapolations from Shafer (Figure 1). With the A02 and A03 data we will be able to make a precise measurement of the fluctuations in the 12-25 $\mathrm{keV}$ band and will have a $2 \sigma$ or better detection in the $35-60 \mathrm{keV}$ energy range.

\section{SYSTEMATICS}

The HEAO-1 A4 study [10] of fluctuations differed from HEXTE in having a much larger beam of $112 \mathrm{sq}$. degrees, smaller area by a factor of four, and complete sky coverage. Suppression of systematics was achieved largely by careful modeling of the detector internal background [16] and tricks of analysis to guarantee cancellation of systematics. The fluctuation measurement was just subtractable (in quadrature) with some confidence from the total variance. Although marginal, it was interesting in indicating a spectral index flatter than the index 1.7 spectrum of nearby bright Seyferts. Since the detector fluctuation level is dominated by sources with a density of a few per beamwidth, the A4 sampled not nearly as deeply as HEXTE does.

The HEXTE was specifically designed with a view to the lessons learned from A4. The aperture switching on a small angular scale and short time scale allows the subtraction of measured background with residual systematics small compared to Poisson errors for observations up to 600000 seconds. We have found systematics to be less than $0.04 \%$ of the internal background at 16 sec- 

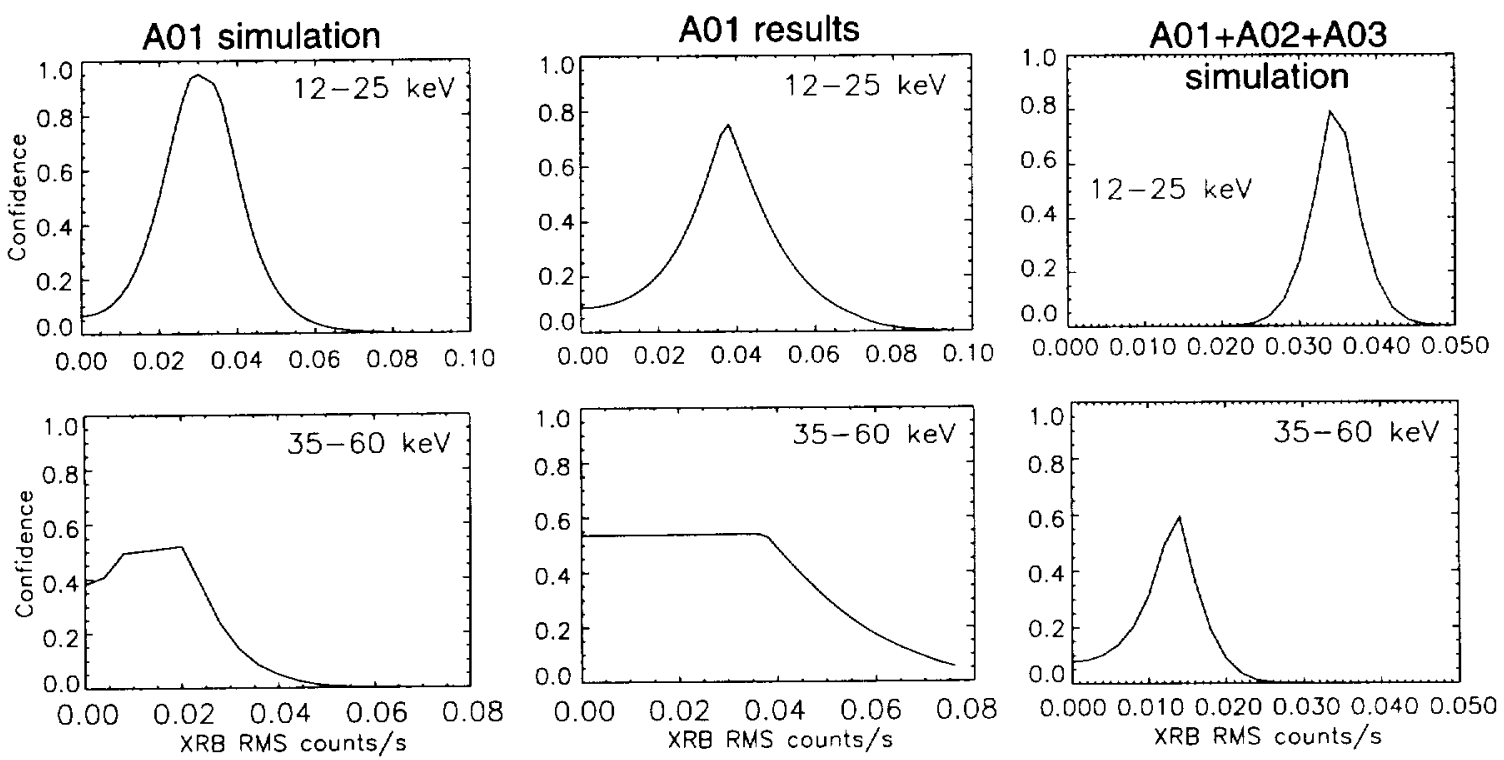

Figure 1. The K-S probability as a function of assumed sky fluctuation noise in units of cluster count rate. (Left panel) monte carlo estimate for the first 9 months (AO1) of the mission, with observation of 60 sources. (Middle panel) Observed results from AO1. (Right panel) estimates for nearly three years of data $(\mathrm{AO} 1+\mathrm{AO} 2+\mathrm{A} 03)$. The $90 \%$ confidence range for the sky noise for $12-25 \mathrm{keV}$ in the middle panel is $[0.020,0.054]$.

onds, which is nominal for HEXTE. Even with the 2 minute dwells of OSSE, one of us (DG) has obtained poisson-limited subtractions in the vicinity of 0.2 percent of internal background. Although internal background can in principal vary with orientation due to the position of the earth and the cosmic-ray east-west effect, no such effect could be measured with $\mathrm{A} 4$, and the 3 or 6 degree modulation for HEXTE should have no uncancelled systematics remotely comparable to the expected signal. Finally, the automatic gain control, to a fraction of a percent, stabilizes the gain wandering that complicated the A4 analysis.

We have divided one very long observation, obtained during A01, into 8 intervals of 100000 seconds each. These are consistent with no variability [17], which is one indication that systematics are so far under control. We can use almost the entire data set to set close limits to the residual errors from subtraction of the time-variable background by employing software selection for differences on longer time scales. Such systematics grow quadratically with the selected difference period.

\section{ANALYSIS METHOD}

As has been done before for the mechanically collimated Uhuru, Ariel-V, HEAO-1 A-2 \& A4 , and Ginga datasets, the analysis of the fluctuations depends sensitively on the solid angle, the number of observations, and the signal to noise. We anticipate that our relative sensitivity in the $20-60 \mathrm{keV}$ band should approach that of the HEAO-1 A-2/Ginga data in the $2-10 \mathrm{keV}$ band and thus be sensitive to 1 source/beam. Using the Madau et al. [5] model this corresponds to a flux limit of $0.2 \mathrm{mCrab}$. Comparison of the implied $\log \mathrm{N}-\log \mathrm{S}$ consistent with the fluctuation analysis will determine the source counts in this band for direct comparison with the $2-10 \mathrm{keV}$ 
source counts.

Comparison of the difference spectrum between the $+2 \sigma$ and the $-2 \sigma$ sources $[18,19]$ should reveal the mean spectrum of sources at the effective confusion limit and thus strongly constrain all the models for the origin of the hard $\mathrm{x}$-ray background. Inclusion of the PCA data for direct comparison will allow a broad band spectrum of these sources to be determined. Comparison of these spectra to HEXTE sources can identify the residual X-ray background with newly measured classes of X-ray sources such as Seyfert 2 galaxies.

Analysis for excess variance among the fields amounts to identification of serendipitous sources, as discussed above, and their exclusion, and then the determination of excess variance from the remaining sample. Since the observations in the sample have different durations, hence sensitivities, we have devised a statistic for the desired quantity, excess variance, based on the Kolmogorov-Smirnoff test. Given the distribution of observing times and an assumed excess variance level in detector count rate units, we synthesize a cumulative distribution function for the set of observations, and compare it with what was observed, and evaluate the K-S probability. This is done for a range of assumed excess variances (see Figure 1), and the maximum of the probability distribution function is taken as the estimator for the best-fit excess variance. Confidence intervals are estimated in the usual way from integrals of this observed distribution function.

\section{RESULTS}

As shown in Figure 1, our best-fit estimate for the sky fluctuation level in the $12-25 \mathrm{keV}$ band from the first nine months of the XTE mission differs from zero by about two sigma, although the width of the $90 \%$ confidence interval is still about a factor of two. It is also close to the amount extrapolated from $2-10 \mathrm{keV}$ measurements [14]. One serendipitous source has been identified, which is at about the expected count. As is shown in Figure 1, the result at this energy from the entire three-year program will be much more tightly constrained, and a useful result is also to be expected in the $35-60 \mathrm{keV}$ band.

\section{REFERENCES}

1. Fabian, A. C., and Barcons, X., Ann. Rev. Astron. Astrophys. 30, 429 (1992)

2. Lightman, A. P. and White, T. R., (1988), ApJ 335, 57.

3. Pounds, K. A., et al., Nature 344, 132 (1990).

4. Nandra, K. and Pounds, K. A., MNRAS 268,405 (1994)

5. Madau, P., Ghisselini, G., and Fabian, A. C., MNRAS 270 L17 (1994).

6. Iwasawa et al. PASJ 46, (1995)

7. Makishima et al., PASJ 46, L77 (1994)

8. Fukazawa et al., PASJ 46, L141 (1994)

9. Carrera, F.J.. et al. MNRAS 275, 22 (1995)

10. Gruber, D. E. in "The X-Ray Background", eds. X. Barcons and A. C. Fabian (Cambridge:Cambridge University Press), p. 44 (1992).

11. Johnson, W. N. et al., in "The Second Compton Symposium", ed C. Fichtel (New York:AIP), p. 515 (1994).

12. Zdziarski, A. A., Johnson, W. N., Done, C., Smith, D., and McNaron-Brown, K., Ap. J. 438 L63 (1995).

13. Madejski, G.M., Zdziarski, A.A.; Turner, T.J.; Done, C.; and others. Ap J, 438, 672 (1995).

14. Shafer, R. A., PhD Dissertation, U. Maryland (1983)

15. Marshall, F. E., et al., Ap. J. 235, 4 (1980).

16. Gruber, D. E., Jung, G. V., and Matteson, J. L., in "High-Energy Radiation in Space" eds A. Rester and J. Trombka (New York:AIP), p 232 (1989).

17. MacDonald et al., BAAS 28 N.4, 1316 (1996).

18. Mushotzky, R. F., et al. in "Frontiers of XRay Astronomy", eds. Y. Tanaka and K. Koyama, (Tokyo:Universal Academic Press), p 657, (1992).

19. Hayashida, K., et al, in "Frontiers of X-Ray Astronomy", eds. Y. Tanaka and K. Koyama, (Tokyo:Universal Academic Press), p 653, (1992). 


\title{
ABS'RRACT OF THE DISSERTATION
}

Measuring the Cimmllarity of the Diffuse Hard X-ray Background

\author{
by \\ Daniel Rufus MacDonald \\ Doctor of I'hilosophy, Graduate program in Physics \\ Universits of California, Riversicle, March, 1999 \\ I'rofessor Allen Zych, Cliairman
}

The diffuse X-ray barkground is widely held to originate largely from distant active galaxies. This view is sulpported by both counts of sources in survey missions and fluctuation studies in the soft $\mathrm{x}$-ray energy band. However, most of the $\mathrm{x}$-ray background emission occurs in the 10-60 keV hard $x$-ray band and sensitive studies in this energy band are only now possible.

The fluctuations in the x-ray background for three wide energy bands have been determined. They are $14.1 \pm 2.0 \%$ of XRB flux from 15.5 to $20.2 \mathrm{keV}, 17.3 \pm 2.6$ \% from 20.2 to $24.9 \mathrm{keV}$ inml $24 \pm 7 \%$ from 34 to $64 \mathrm{keV}$ for a 1.1 square degree field of view.

During normal observil ions, the High Energy X-ray Timing Experiment instrument (HEXTE) on the liusi X-ray Timing Explorer (RXTE) Spacecraft makes 
nearly simultane degrees. Differenc's in fluxes from these pairs of background fields can be used to detect a source poumlation much deeper than can be studied from individual detections. A measurencint of the fluctuations in the X-ray background in wide energy bands above $15 \mathrm{k}$ (an be made using the ensemble of all long RXTE pointings, a total of $\sim 36.7$ millin seconds of observations. A distribution of the sky flux differences can be mark from a data set of these long pointings. Tails in the distribution of the differences are dominated by sources that occur at a spatial density of one source per field of view. The nominal HEXTE instrument field of view is one square degree, but this linll (an be smeared out by scasonal rotation of the spacecraft. An excess variatioi! an be found by compriting the the fluctuation distribution to a distribution chincterized by a data set that only contains statistical counting errors. This exces variation in wide energy bands is used to create the fluctuation spectrum. 